\title{
Exploración de materiales y técnicas de fabricación digital y prototipado rápido para el diseño y desarrollo de recursos didácticos de apoyo a la enseñanza del color en la etapa escolar
}

Exploration of materials and techniques for digital fabrication and rapid prototyping for the design and development of didactic resources to support the teaching of color in school environments

\author{
Ingrid Calvo Ivanovic \\ Departamento de Diseño, Universidad de Chile \\ ingridcalvo@uchilefau.cl \\ - Bruno Perelli Soto \\ Departamento de Diseño, Universidad de Chile \\ bperelli@uchilefau.cl
}

\begin{abstract}
This project arises from the need to produce didactic resources to support the teaching of color in the Chilean school environment. It starts from a critical screening at the Visual Arts curriculum proposed by the Ministry of Education. The way to address this lack of resources was through exploration techniques of digital fabrication and rapid prototyping, in order to generate updated and low cost learning objects. This could provide didactic materials to a wider amount of teachers and learning environments. The prototypes created will be implemented in the Centro de Arte y Tecnología, of the Fundación Mustakis, a pioneering space for independent learning and exploration around chromatic topics.
\end{abstract}

Keywords: Color, Educación, DIY, Fabricación Digital, Arte Interactivo

\section{Introducción/Contexto}

El color es un recurso expresivo fundamental de la experiencia estética. Como potente catalizador de emociones y sensaciones, estimula la sensibilidad y la creatividad de los seres humanos desde su desarrollo cognitivo temprano. Por esta razón, el aprendizaje del color ha sido considerado en los programas de estudio de las escuelas chilenas, en las asignaturas de carácter artístico, desde el siglo XX. Su enseñanza formal ha estado históricamente marcada por el reconocimiento de conceptos ligados a la "Teoría del color", cuyos principales planteamientos datan desde siglo XVI hasta principios del siglo XX. El aprendizaje teórico del color es el aprendizaje de una técnica (Calvo, 2009), el sometimiento de ésta a ciertas leyes, cuyo reconocimiento permite manejar los medios más adecuados para expresar ciertos colores, evitar la monotonía de las combinaciones, estimular el gusto selectivo no azaroso y afirmar la sensibilidad.

Sin embargo, en un estudio de estado del arte de la enseñanza formal del color en las escuelas chilenas realizado en 2015 (Calvo, 2015), que contempló una revisión a los contenidos y metodologías empleados para enseñar el uso del color en los programas de Artes Plásticas definidos por el Ministerio de Educación de Chile para la Enseñanza Básica y Media, se constata que la actual inclusión de esta materia en el currículo presenta notorias deficiencias tanto en cantidad de horas dedicadas en el aula como en la calidad epistemológica de los contenidos enseñados, lo que se evidencia en la notoria desactualización de las bibliografías empleadas y la carencia de recursos y materiales didácticos -tanto análogos como digitales- para apoyar la enseñanza de dichos contenidos. Este último punto puede deberse a la escasez de insumos didácticos para esta finalidad producidos localmente (probablemente por el alto costo de producción, impresión y/o coloración de los materiales empleados) y a la especificidad y elevados costos de importación de recursos didácticos producidos fuera del país, como es el caso de los modelos para la enseñanza del color Natural Color System (NCS) o Munsell.

A partir de lo anterior, el objetivo del presente proyecto es explorar materiales y técnicas de fabricación digital y prototipado rápido para diseñar y desarrollar prototipos de recursos didácticos para la enseñanza del color en el aula, que apoyen el aprendizaje interactivo del color en las asignaturas relacionadas con la educación artística de la etapa escolar en sus niveles Básica y Media. Además este proyecto tendrá como visión una política de accesibilidad, buscando su desarrollo mediante la tecnología y medios más idóneos para que los prototipos se generen dentro de un margen de producción de bajo costo para los usuarios y, de esta manera, otorgar un mayor acceso a los recursos didácticos propuestos.

Los prototipos de recursos didácticos que surgen a partir del desarrollo del presente proyecto serán testeados en el futuro Centro de Arte y Tecnología de la Fundación Mustakis (http://www.fundacionmustakis.com/), que se inaugurará en 
enero de 2016, y constituirá un espacio para el aprendizaje extracurricular de experiencias artísticas por parte de niños y jóvenes. Este Centro contemplará la creación de una Sala Color, que en su primer período (año 2016), abordará la temática central de "Color y luz" y buscará suplir parte de las carencias ya declaradas de los programas formales de educación artística, a la vez que contemplará la inclusión de experiencias interactivas, un entorno de trabajo con talleres y la implementación de material didáctico para la enseñanza del color.

Adicionalmente, este contexto de testeo, implica la necesidad de que los materiales y objetos didácticos potencien la experiencia de auto-aprendizaje interactivo y autónomo, donde los estudiantes puedan manipularlos sin la ayuda de un monitor o profesor, y donde a través de su propia exploración (a veces incluso contribuyendo a la creación de sus propios materiales, como círculos cromáticos u otros) puedan aprehender los contenidos más relevantes para la observación y aplicación de los colores.

\section{Metodología}

Como se ha planteado, este proyecto proveerá de material didáctico a la Sala Color, en el marco de la temática general "Color y luz", donde la relación del color y la luz será entonces el punto de partida didáctico y experimental que articulará el aprendizaje del color en todas sus dimensiones. Esta relación -entre color y luz- es comúnmente la primera manera de acceder a la comprensión del fenómeno cromático: comprender al color implica experimentar con la luz. La relación entre el color y la luz cumple un papel fundamental para mantener la dialéctica entre razón y emoción, entre sentimiento e intelecto, y a través de ello, la experiencia de la armonía y la belleza (Tornquist, 2008). La relación entre color y luz será también el lugar desde donde se proyectarán las experiencias y recursos didácticos propuestos.

\section{Diagnóstico - resultados análisis del currículo escolar chileno, asignaturas de Artes Visuales}

Se revisaron los contenidos relacionados con la enseñanza del color en cada uno de los programas de estudio de la asignatura de Artes Visuales dispuestos por el Ministerio de Educación para los 8 niveles de Enseñanza Básica y los 4 niveles de Enseñanza Media, con la finalidad de analizar qué se enseña y cómo son presentados estos contenidos a los estudiantes. También se hizo una revisión al material didáctico de apoyo digital, descargable de la web del Ministerio de Educación (MINEDUC).

Conclusiones generales a partir de la revisión de planes y programas de enseñanza básica y media:

a. La utilización de conceptos teóricos específicos asociados al color se encuentra casi en su totalidad desactualizada, se recomienda encarecidamente una revisión profunda en función de bibliografía actualizada, la corrección de errores y la precisión de algunos conceptos. Se constata que la situación de la enseñanza del color en el currículum de Artes Visuales es dramática y precisa de cambios urgentes y generación de nuevas instancias o materiales de apoyo que complementen la educación artística en este aspecto.

b. Se explora muy poco la relación de los colores con la luz, la cultura latinoamericana o chilena, la relación del color con la identidad o el uso de los colores para representar mensajes. No se enseña a comunicar con color, más allá de una revisión leve a la relación del color con las emociones, aunque sólo desde una aproximación intuitiva y predisponiendo al estudiante a la asociación de determinados colores con sentimientos o emociones.

c. En los niveles de $7 \mathrm{mo}$ y $8 \mathrm{vo}$ básico se proponen actividades destinadas a analizar algunos movimientos artísticos, tales como el fauvismo, cubismo y el neoplasticismo, entre otros. Sin embargo en los programas de ambos niveles el color no aparece con contenidos específicos asociados, y el alcance del análisis que el estudiante puede realizar aparece desaprovechado, al no entregarle las herramientas necesarias para hacerlo.

d. El material didáctico de apoyo digital, descargable de la web del Ministerio de Educación a los programas de Artes Visuales de enseñanza básica es insuficiente: en casi todos los niveles se limita a unas pocas diapositivas, con deficiente uso de recursos gráficos y de imagen. Por su parte, no existen recursos didácticos de apoyo descargables para los 4 niveles de enseñanza media, a pesar de que en 1ero medio existen contenidos específicos asociados al color.

e. En los niveles de 7mo y 8vo básico, además de 1ero medio, se propone por parte del docente algunos análisis cromático más profundo, principalmente a obras y movimientos artísticos. Sin embargo, estos ejercicios de análisis son propuestos a partir de la suposición de que existen determinados conceptos aprehendidos por parte del estudiante, los cuales no habían sido incorporando en etapas previas del currículo. Escasez de herramientas para realizar los ejercicios correctamente.

f. Prácticamente a los estudiantes en todos los niveles de la enseñanza escolar no se les otorgan las herramientas para aprender a combinar los colores. El concepto de armonía fundamental en este aspecto- no parece asociado a ningún tipo de ejercicio (sólo aparece mencionado en un glosario) y el concepto de contraste aparece escasamente desarrollado e incluso, en ocasiones, aparece mal empleado.

g. Como ha sido posible constatar, la enseñanza del color se va progresivamente eliminando del currículo desde el 1ero básico hasta el 6to básico. Desde 7mo básico en adelante -con excepción de algunos ejercicios de 1ero medio- el color no aparece con contenidos específicos asociados en los programas de educación artística. Lamentablemente, es precisamente en estos niveles superiores donde este recurso podría ser objeto de análisis y ejercitación más profunda y significativa, ligándose a conceptos de identidad nacional, barrial, personal y cultural. Este aspecto impacta profundamente en la educación superior, en disciplinas proyectuales relacionadas con el Arte, 
el Diseño y la Arquitectura, por ejemplo, donde de no existir asignaturas específicas sobre Color (lo que generalmente sucede en esos programas de estudio), la enseñanza formal en torno a materias de color queda prácticamente supeditada a lo aprendido hasta 6to básico.

\section{Análisis de referentes de recursos didácticos}

Luego del análisis al currículo escolar, fue necesaria una revisión de referentes y recursos didácticos existentes que exploran la relación del color y la luz. Cabe señalar que ninguno de los recursos analizados puede encontrarse en la actualidad en el mercado chileno -lo que denota la escasez de recursos y dificultad de acceso a los existentes, antes señaladas-, por lo que éstos sólo pueden conseguirse mediante compra electrónica internacional. Algunos de los recursos analizados son:

Jonti-Craft, Tabletop Light Box: Es una mesa de luz portátil retroiluminada, que permite el aprendizaje y exploración con diferentes juegos y materiales educacionales, de carácter translúcido. Permite explorar la mezcla óptica o síntesis aditiva del color a partir de la interacción de la luz con el color, a partir de la superposición de figuras geométricas. Materiales: cubierta de acrílico, sistema eléctrico de paneles reflectantes, estructura de madera y goma antideslizante, figuras geométricas de acrílico de color.

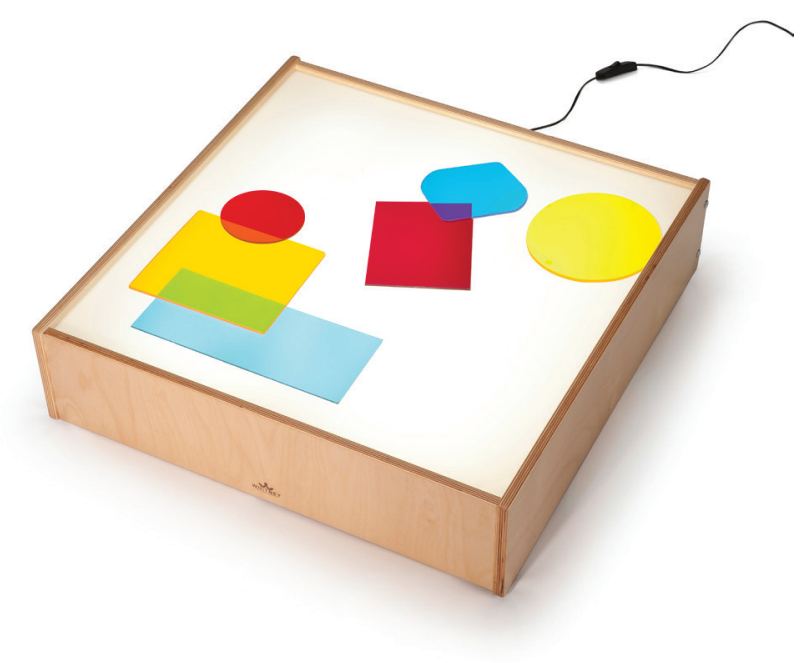

Figura 1: Tabletop Light Box.

Naef Spiele, Bauhaus Optical Mixer of Colors: Este juguete muestra cómo se produce la mezcla óptica de colores mediante la rotación de discos. Basado en la teoría de mezcla a través de discos giratorios de Isaac Newton, las plantillas circulares intercambiables permiten visualizar distintos aspectos del color, propuestos por teóricos como Goethe, Schopenhauer y Hölzel. Este objeto es parte de una serie de réplicas de juguetes diseñados a principios del siglo XX en la escuela Bauhaus. La fábrica Suiza de juguetes de madera Naef Spiele bajo licencia Bauhaus produce estas réplicas a partir de los dibujos originales encontrados en los archivos de la escuela. Materiales: estructura cónica y mango de madera, 7 discos de cartón impresos en offset 4/1.

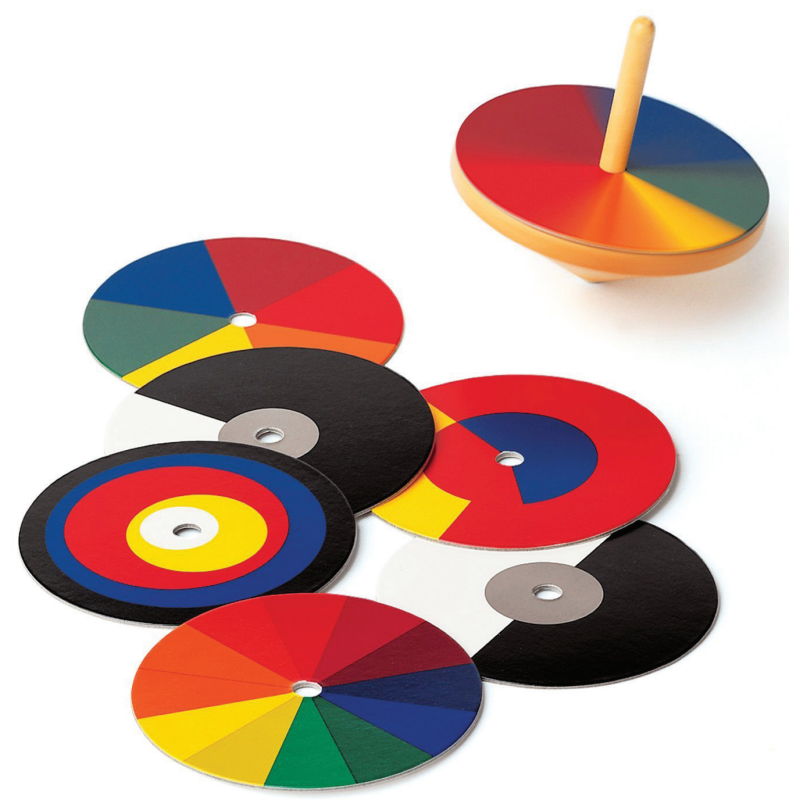

Figura 2: Bauhaus Optical Mixer of Colors.

LR Learning Resources, Color mixing glasses: Anteojos que exploran la mezcla óptica del color a partir de la superposición de 2 filtros coloreados translúcidos en forma simultánea. Se detecta en el análisis de este objeto que la idea propuesta podría aprovecharse de mejor manera si se permitiera la superposición de 3 filtros simultáneos (en vez de dos) y si existiera mayor control o cuidado del color de los filtros, de manera de asegurar que los colores básicos son los colores primarios de la luz (rojo, verde y azul) y no colores al azar. Materiales: estructura de polipropileno coloreado azul, filtros coloreados de acrílico.

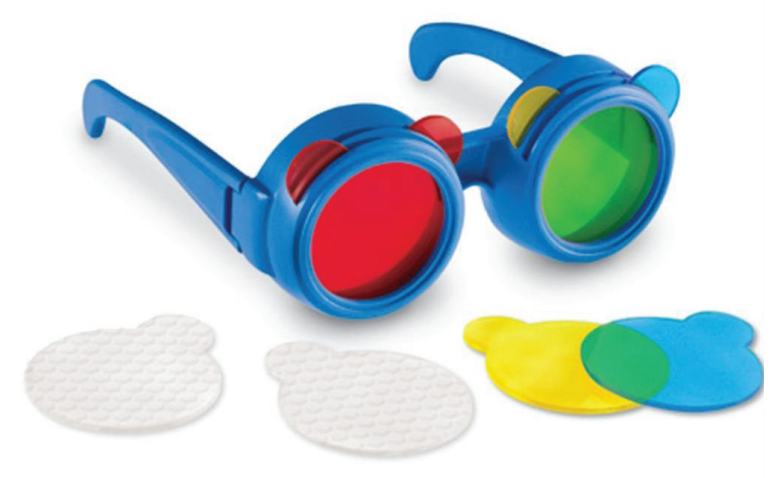

Figura 3: Color mixing glasses. 
Rueda de color CMY: Consiste en 3 círculos concéntricos con troqueles circulares ordenados en forma espiral. Los círculos se encuentran unidos por un eje central que permite su rotación y fijación a superficies a partir de un mecanismo de succión. Cada círculo está hecho de papel vegetal coloreado, correspondiente a los tres colores de la síntesis sustractiva, cian, magenta y amarillo. Al fijar el sistema en una superficie que permita el paso de luz, como una ventana, y rotar los círculos, es posible experimentar con la aparición de nuevos colores mediante la superposición de las capas coloreadas. Materiales: círculos de papel vegetal troquelado, dos capas de polipropileno de $1 \mathrm{~mm}$. para protección, mecanismo central de succión.

La obra de Floor Van de Velde: tridimensionalidad, retroiluminación de superficies, planos seriados y control de luz. El trabajo realizado por esta artista plástica Sudafricana, contempla la mezcla de recursos fotográficos, sonoros y lumínicos sobre una variedad de formatos, algunos de los referentes tomados, destacan por la utilización de materiales como acrílicos y la retroiluminación de éstos a través del uso de LEDs.

\section{Diseño de propuestas de material didáctico}

A partir del levantamiento de recursos existentes y su análisis, es posible señalar que en la exploración didáctica del color en su relación con la luz, es necesario recurrir a algunas metodologías de recreación de ilusiones y experimentos ópticos tales como la iluminación, la retroiluminación, la translucidez y la rotación. Estas metodologías resultaron claves para el diseño de los recursos didácticos del presente proyecto.

Los objetos didácticos propuestos son:

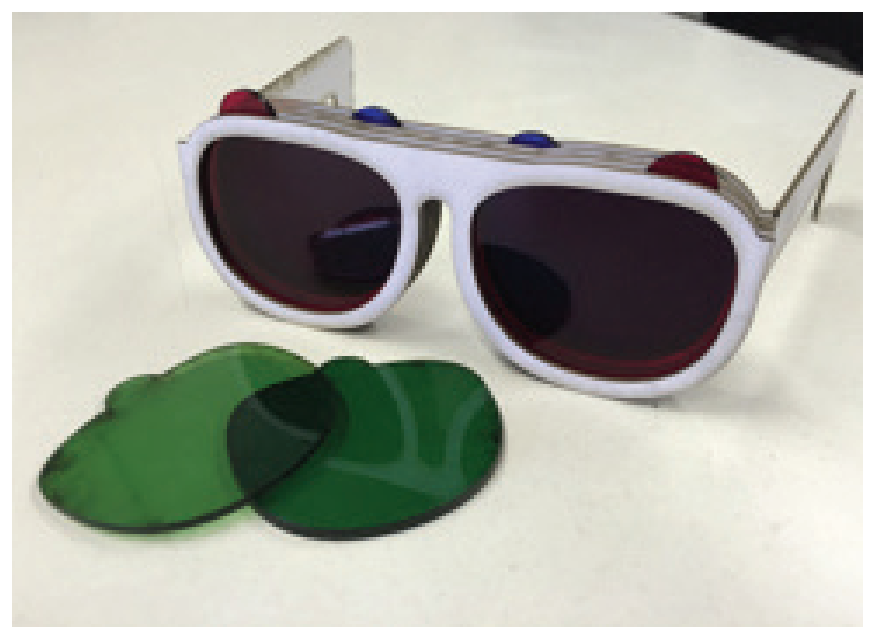

Figura 4: Lentes aditivos.

Lentes Aditivos: Para la realización de este prototipo se consideraron las metodologías didácticas de translucidez y retroiluminación. A partir del referente analizado (Color mixing glasses) se propone una reinterpretación desde el diseño y el contenido, hacia el mejoramiento de ese objeto didáctico. El prototipo "Lentes aditivos" consiste en unos anteojos que permiten la superposición de capas de acrílico translúcido coloreado con los matices de la síntesis aditiva o mezcla de luces, para una correcta comprensión del fenómeno cromático en su relación lumínica. Además, y según la teoría cromática contemporánea, la mezcla siempre surge a partir de una tríada de colores primarios, por lo que esta reinterpretación del objeto didáctico contempla la posibilidad de trabajo con tres filtros de color para cada ojo, mejorando a su antecesor.

Run Run Cromático: Para este prototipo, se consideró la metodología didáctica de rotación de materiales coloreados. El "run run" es un juguete tradicional de gran parte de los países de Latinoamérica y consiste en una pieza circular con dos orificios a igual distancia de su centro (comúnmente un botón), por los que atraviesa un cordel formando lazo alrededor de este. Su nombre proviene del sonido que este objeto genera al tirar el cordel enroscado y rotarlo en torno a su eje. En el prototipo propuesto, ambas caras del círculo contemplan un círculo cromático de 6 colores, que al girar genera el fenómeno de la mezcla óptica.

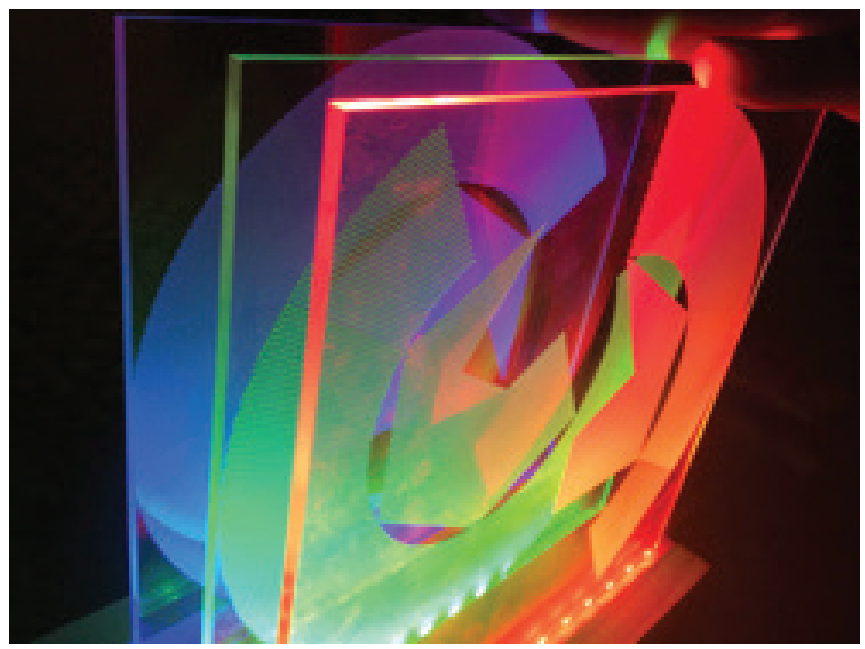

Figura 5: Rueda de Color LED.

Rueda de Color LED: En este caso, se contempló la metodología didáctica de iluminación. Se consideró la problemática que se plantea comúnmente, ya que se requieren pantallas para recrear condiciones que expliquen la interacción entre canales de color luz (RGB) para la generación de otros colores. La finalidad de este prototipo es la generación de un modelo didáctico de exploración del color luz, que sea tangible y que no siempre signifique la interacción con dispositivos digitales. El referente utilizado es la Rueda de Color de Johannes Itten (Itten, 1961), un círculo cromático planteado a partir de doce colores o matices. $\mathrm{Si}$ bien no fue pensado para la representación de luz, establece combinatorias controlables en este ámbito. Se procedió a separar la imagen de Itten en tres canales: rojo, verde y azul. El 
resultado de esta separación son imágenes en escala de grises, como tramas base para la captura del color. Algunas pruebas preliminares habían demostrado que el grabado de imágenes raster con láser atrapan la luz sobre la superficie grabada. Para generar una combinatoria de luz, se dejan espacios sin trama en el acrílico, que serán los lugares donde se produzcan las mezclas ópticas.

\section{Prototipado y exploración material}

Lentes Aditivos: Para la generación de este prototipo se consideraron tres posibles caminos; primero, se consideró la evaluación de los materiales más idóneos para las lentes de color. Un testeo preliminar, evidenció que la utilización de materiales como micas de color o papeles coloreados semi-translúcidos eran opciones no viables puesto que estructuralmente presentan problemas, ya que al someterlos al calor del corte láser, tendían a curvarse o estropearse. Por esta razón, el equipo optó por la utilización de acrílico, material rígido y apto para el corte con láser. El segundo criterio de desarrollo fue la generación de los marcos de los lentes; el producto original contaba con marcos plásticos rígidos que podrían haber sido recreados utilizando una impresora 3D, sin embargo, la idea de generar un producto de fabricación "low cost", desechó la idea de desarrollar el producto con esta tecnología de fabricación digital. Para la maqueta inicial se decide entonces utilizar cartón de 1,5 mm de espesor, y para el prototipo final, madera. La idea fue la implementación de planos seriados en las cuales se intercalaron los acrílicos, permitiendo la obtención de colores nuevos mediante mezcla óptica.

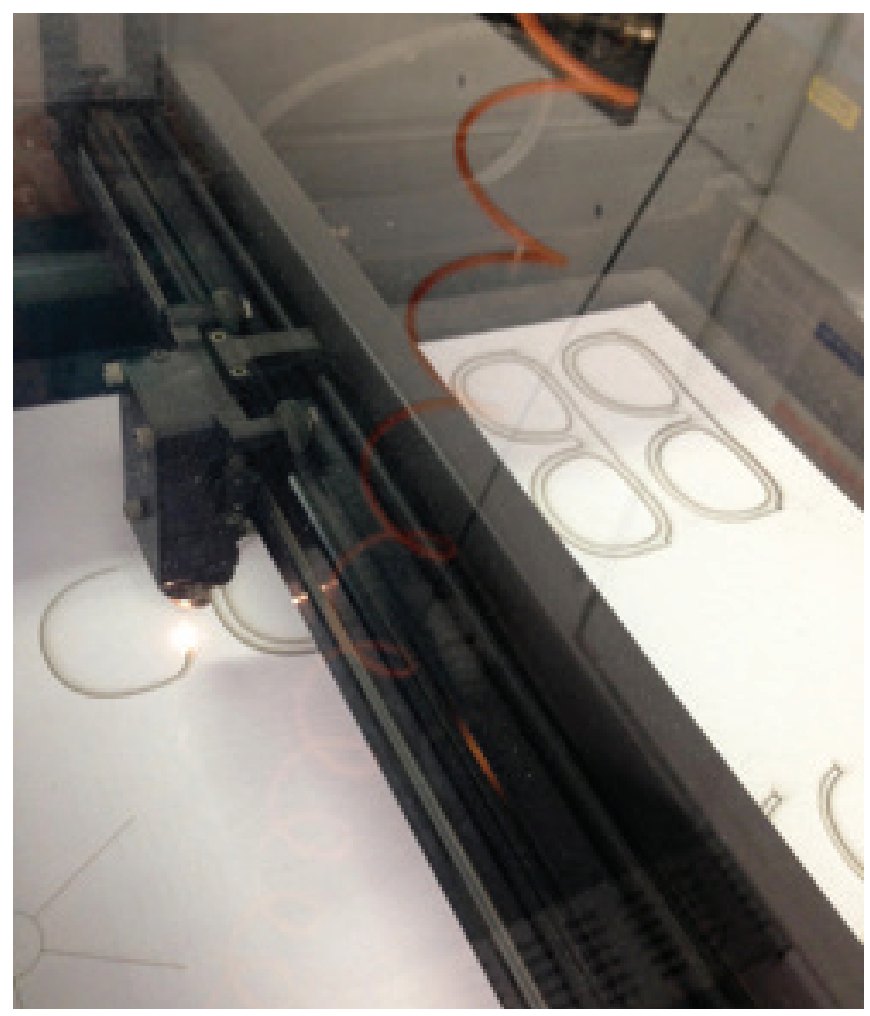

Figura 6: Corte laser de piezas de Lentes Aditivos.
Run Run Cromático: En un principio, se buscó la manera más sencilla de generar un objeto didáctico para el aula, que los mismos estudiantes fueran capaces de generar, optando por la realización de un disco de cartón al cual se adosarían papeles de colores. Sin embargo, la opacidad propia del papel coloreado o teñido no lograba la intensidad o saturación de color deseada. Es por esta razón que se decidió cambiar el material a vinilo autoadhesivo, ya que este material al ser un polivinilo, tiene un color mucho más definido y estable. En este caso, se utilizó el corte láser para el troquelado y grabado de los discos de cartón, pero no se consideró como opción para el vinilo, debido a que la temperatura del láser deformaría el material. Para las piezas coloreadas se optó entonces por utilizar plotter de corte el cual funciona igual que una impresora, salvo que su cabezal no deposita tinta, sino que tiene una hoja afilada que corta el vinilo. Finalmente, la propiedad de ser autoadhesivo por una de sus caras, hace que la integración con el disco de cartón (espesor 1,5 mm), sea mucho más limpia.

Rueda de Color LED: Este prototipo aborda la exploración de materiales y procesos de fabricación digital, en su vinculación con saberes tradicionales de impresión y el manejo del color en su relación con la luz. Para lograr la superposición de colores aditivos primarios, rojo, verde y azul, se controló la intensidad de cada color; luego por mezcla se originaron los matices secundarios, recurriendo a técnicas de impresión como las tramas de semitono. Siguiendo estas técnicas de impresión, los canales de colores rojo, verde y azul se tramaron en diferentes ángulos, y se logró asegurar la presencia del color en forma dosificada y por otra parte, la generación de espacios blancos para la aparición de los otros dos matices primarios. Las imágenes grabadas en el acrílico, se trabajaron digitalmente a 1 bit de información, para evitar la contaminación de tonos negros, en un formato de manejo ideal para bitmaps (BMP). A continuación, se retroiluminó cada placa de acrílico con LEDs de alto brillo de los colores primarios ya señalados, rojo, verde y azul.

La elección de la tecnología de luces LEDs respondió a la necesidad de trabajar el color de manera controlada. Las zonas de acrílico sin grabar generan el rebote de luz entre las paredes del material, mientras, las zonas grabadas atrapan la luz permitiendo su fijación en la superficie del acrílico, y dando lugar a las zonas coloreadas. La utilización de tramas de semitono garantizan esta fijación del color en puntos de color luz, y una serie de superposiciones de las tramas grabadas en porcentajes, posibilitó la generación de nuevos colores mediante porcentajes de luz.

\section{Resultados}

En su primera versión, de prototipo o maqueta, los materiales han sido puestos a prueba, obteniendo experiencias que deben ser evaluadas y corregidas para las versiones finales. A partir de esto, los resultados obtenidos de la interacción con los prototipos son las siguientes:

Lentes Aditivos: Si bien, el modelo original está basado en 
un juguete comercial, se probó y evaluó para la realización de correcciones en la cantidad de lentes intercambiables y la elección de los matices idóneos para la aplicación de síntesis aditiva de color, objetivo del recurso didáctico. Una vez ensamblado el prototipo, se advirtió que la síntesis se lograba de manera parcial debido a la intensidad de los colores propios de los acrílicos utilizados, en particular los que corresponden a rojo y azul, cuyos matices son bastante más saturados que el filtro verde, destacando por sobre ese tercer color y opacándolo, impidiendo la obtención del efecto deseado. Sin embargo, la superposición de los filtros rojo y azul logra generar la correcta síntesis aditiva, generando el matiz magenta.

En una etapa posterior, se evaluará trabajar con un espesor de acrílico inferior al actual de dos milímetros, de manera que la luz que se filtre de manera más eficiente.

Run Rún Cromático: De los tres prototipos elaborados, el run rún es el objeto didáctico que demostró una mayor eficacia en el traspaso de los contenidos a los usuarios, ya que al momento de experimentar con los colores, la estabilidad cromática aportada por los vinilos autoadhesivos logró generar correctamente la síntesis aditiva por rotación. La ventaja de introducir este juego tradicional, reconocido por la mayoría de los niños chilenos, garantiza su correcto uso y, por ende, la comprensión de las materias por parte de los estudiantes. Se anticipa que la interacción con este objeto, será la que generará mayor atención en los destinatarios.

Rueda de Color LED: La combinación de tramas de semitonos con técnicas de fabricación digital logró una efectiva generación de nuevos colores mediante la superposición de las placas de acrílico. Sin embargo, se recomienda que la retroiluminación LED se produzca internamente en el acrílico, de forma tal que se aumente el rebote de luz al interior de dicho material, además de evitar que se generen espacios entre las placas de color, producto de la utilización de bandas LED. El espesor utilizado en los acrílicos para esta experiencia, fue el adecuado (5 milímetros), debido a que un grosor inferior no permitiría que el LED se inserte al interior del canto del material.

Los testeos en torno a los prototipos, ya evaluados, permitirán realizar las mejoras pertinentes para una aplicación más efectiva de estos materiales dentro de escuelas y colegios en Chile, a través de la red de conexiones de la Fundación Mustakis y su programa Colorearte.

\section{Conclusiones}

Se evidencia que la elaboración de materiales didácticos que potencien la enseñanza de materias cromáticas supone un gran desafío debido, principalmente, al carácter relativo del color como recurso plástico y expresivo, y su constante cambio en la intrínseca relación con las condiciones lumínicas. Dicha relatividad se hizo presente durante el proceso de fabricación de prototipos, en los cuales el equipo investigador debió privilegiar ciertos materiales por sobre otros, en favor de una mayor estabilidad del color, dadas las diversas condiciones de la experiencia a realizar.

En adición a lo anterior es posible señalar que en toda actividad de diseño y desarrollo en torno al color debe considerarse un entorno controlado, idealmente estandarizado según metodologías de control de calidad de color, poniendo atención a las condiciones lumínicas, la colorimetría de los materiales en función de lograr el mejor rendimiento en la apariencia visual, y las condiciones del observador, emulando los entornos de aprendizaje donde los materiales serán utilizados. Sin embargo, el control del color requiere de instrumentos ópticos y tecnologías de medición las que, lógicamente, encarece el proceso de diseño y desarrollo de los prototipos, alejándose de los objetivos planteados por el presente proyecto.

Con respecto al rol de las técnicas de fabricación y prototipado, queda demostrada su versatilidad y utilidad, sobre todo en la intervención con materiales de bajo costo, tales como acrílico y cartón, utilizados durante la elaboración de los objetos didácticos. Por otro lado, la vinculación de técnicas propias del mundo de la impresión como la generación de tramas de semitonos con técnicas de fabricación digital, en el caso del objeto didáctico Rueda de Color LED, demostró un camino interesante para la generación del efecto de síntesis aditiva, que puede explorarse más en una etapa posterior del proyecto.

Como equipo investigador creemos que es importante abordar con mayor detenimiento la implementación de otros materiales y técnicas para la interacción con color y luz. $\mathrm{Si}$ bien, durante este proceso hemos explorado materiales tradicionales de bajo costo, utilizados comúnmente en laboratorios de fabricación, pensamos que existe un nicho por explorar en la utilización de materiales no convencionales que mejoren la experiencia de control, percepción y comprensión del color en su relación con la luz.

\section{Trabajo Futuro}

Las siguientes etapas, consideran un proceso de evaluación y correcciones de los prototipos generados, en la cual la validación con usuarios es importante. Es por esto que, como equipo, hemos establecido que el trabajo a futuro considere las siguientes etapas:

1. Presentación de Prototipos a Fundación Mustakis.

2. Exploración de nuevos materiales no considerados durante etapas preliminares.

3. Pruebas con Usuarios en colegios.

4. Desarrollo de otros prototipos en relación con los contenidos propuestos para la Sala Color.

\section{Agradecimientos}

Nuestros agradecimientos a la Fundación Mustakis por motivarnos a pensar en el color, desde su didáctica y experimentación material. 


\section{Referencias}

Calvo, Ingrid. (2009). Proyectacolor, recursos de apoyo a la manera tradicional de estudiar y enseñar el color para el diseño. Proyecto para optar al título de Diseñadora, Mención Gráfica. Santiago: Universidad de Chile.

Calvo, Ingrid. (2015). Informe de estado del arte: Recursos para la enseñanza y aprendizaje del color. Santiago: Fundación
Mustakis.

Itten, Johannes. (1961). The art of color; the subjective experience and objective rationale of color. New York: Reinhold Pub. Corp.

Tornquist, Jorrit. (2008). Color y luz, teoría y práctica. Barcelona: Editorial Gustavo Gili. 